\title{
Radiological significance of high-resolution computed tomography for elderly pulmonary tuberculosis patients - an analysis with culture test
}

\author{
Niranjan Sahu ${ }^{1 \mathrm{PAEF},}$, Somadatta Das ${ }^{2 B, E}$, Rabindra Nath Padhy ${ }^{2 C, D}$ \\ 'Department of Radiology, Institute of Medical Sciences and SUM Hospital, Siksha 0 Anusandhan (Deemed to be University), Bhubaneswar, India \\ ${ }^{2}$ Central Research Laboratory, Institute of Medical Sciences and SUM Hospital, Siksha 0 Anusandhan (Deemed to be University), Bhubaneswar, India
}

\section{Abstract}

Purpose: Pulmonary tuberculosis (PTB) is one of the major health problems in the elderly population, causing significant morbidity and mortality. The aim of this study is to evaluate the significance of the high-resolution computed tomography (HRCT) modality for the diagnosis of PTB, in comparison to culture test.

Material and methods: Thoracic HRCT images of the study population, comprising 124 patients clinically suspected for PTB with smear and culture reports, were analysed for sensitivity and specificity of the HRCT test. Features of active PTB were centrilobular nodules, 'tree-in-bud' pattern densities, macro-nodules, consolidations, cavitary lesions, ground-glass opacities, and miliary nodules.

Results: Among the study population, 108 cases presented HRCT features of active PTB and the remaining cases were negative but had presented a few features mimicking PTB. As inferred from positive culture test results, 106 cases had active PTB, the remaining cases were culture negative for PTB. False-positive (FP) or 'type I error' cases, and false-negative (FN) or 'type II error' cases were ascertained by Bayes' theorem. Sensitivity (true positive rate) and specificity (true negative rate) of HRCT test were 0.8125 and 0.8571 , respectively.

Conclusions: For proper diagnosis the predictive capability, as two values of 'a posteriori probability', was computed; the mean value of 'a posteriori probability' for HRCT was 0.6358 . When its culture test was positive, the HRCT test was $69.56-92.85 \%$ efficient in ascertaining positive results with a sample; on the other hand, when its culture test was negative it was $66.66-100 \%$ efficient for a negative result. Thus, the HRCT test is considerably dependable.

Key words: pulmonary tuberculosis, elderly population, high-resolution computed tomography, culture test.

\section{Introduction}

Pulmonary tuberculosis (PTB) caused by Mycobacterium tuberculosis is a common health problem in the elderly population with the presence of several predisposing factors such as poverty, crowded living conditions, and lack of disease awareness. Obviously, the inability to give an accurate account of symptoms is an underlying problem among the elderly, which contributes to the late recognition of the disease. Moreover, associated chronic co-morbidities alter the presentable clinical picture, leading to delayed diagnosis and aiding in advancement of the disease. The diagnosis of active PTB on high-resolution computed tomography (HRCT) is based on the pattern of parenchymal involvement with features of endo-bronchial spread in the form of centrilobular nodules and 'tree-inbud pattern' densities [1]. Because conventional radiography cannot ordinarily detect the above features of active infection, HRCT has become the modality of choice for diagnosis of active PTB as well as for detection of subtle cavitary changes in large pneumonic consolidations and dense nodular lesions [2]. Due to higher frequency of tu-

Correspondence address:

Somadatta Das, Central Research Laboratory, Institute of Medical Sciences \& SUM Hospital, Siksha 0 Anusandhan (Deemed to be University), K8, Kalinga Nagar,

Bhubaneswar, Pin-751003, 0disha, India, e-mail: somadatta90@gmail.com

Authors' contribution:

A Study design · B Data collection · C Statistical analysis · D Data interpretation · E Manuscript preparation · F Literature search · G Funds collection 
berculosis (TB) related mortality in elderly patients, maintenance of a high index of clinical suspicion for PTB in this vulnerable population is undoubtedly justifiable [3].

Bayes' theorem is used for analysing the available data to assess the role of the HRCT test as a preferential imaging modality for diagnosis of PTB. This analysis quantifies cryptic errors in this coveted test method used for diagnosis of active PTB infections. Thus, the error of treating false-positive (FP) or 'type I error' (HRCT-positive, culture-negative for PTB) cases with anti-tubercular therapy, which looms large to a patient normally, as well as the consequent spate of non-target treatment complications, could be avoided. Moreover, false-negative (FN) or 'type II error' (HRCT-negative, culture-positive for PTB) cases should not be neglected, because the chance of disease aggravation is obvious at the elderly stage. True positives (TPs) (HRCT-positive, culture-positive for PTB) and true negatives (TNs) (HRCT-negative, culture-negative for PTB) are the coveted results of a correct diagnosis. HRCT modality through a set of test statistics was compared with the gold standard culture test. The HRCT test may appear less reliable than the culture test, in the face of a few other diseases often presenting features mimicking PTB. Thus, an all-out analysis of both of these diagnostic test methods for active PTB infections is essential for assessment of their diagnostic accuracy using Bayes' theorem, but because that is unavailable in the literature, this work is anticipated to strengthen confidence levels on these tests.

\section{Bayesian analysis}

The concept of Bayesian analysis regarding the accuracy of a test method is vividly described [4]. The area under receiver operating characteristic (ROC) curve, or area under the ROC curve (AUC), a graphical analysis drawn with values of sensitivity and specificity for diagnostic efficiency, allows estimation in parallel to the predictive capability of a posteriori probability, apart from the arithmetic computation for a posteriori probability as a post-test analysis of the data [5].

\section{Material and methods}

The study population comprised of 124 elderly patients (82 males) aged over 65 years, had attended the Radiology department for HRCT test of the thorax during September 2015 and August 2019. The abovementioned patients clinically suspected for PTB with sputum smear and culture test reports for Mycobacterium tuberculosis, were included under the study population. Thoracic HRCT images of the study population were retrospectively analysed and were grouped into two cohorts: 1 . Patients having typical HRCT features of active PTB infection (HRCT-positive); and 2. Patients not having any HRCT finding of active PTB (HRCT-negative), but presenting a few HRCT features mimicking PTB infection. The main objectives were to evaluate the diagnostic accuracy of the HRCT test in the differentiation of the abovementioned two cohorts of patients, and to analyse the sensitivity and specificity of HRCT test in comparison to sputum culture results for PTB. The accuracy of the HRCT test can be measured by comparing it with the results of culture test, the gold standard test, because it reveals the true status of PTB disease.

Typical HRCT features for PTB were centrilobular, macronodular, and branching linear and/or nodular ('tree-in-bud' pattern) parenchymal densities. The few other findings were consolidations (lobular, subsegmental, segmental or lobar), cavitary lesions (single or multiple), ground glass opacities, and miliary nodules. Associated HRCT features such as, mediastinal adenopathy, pleural effusion, fibro-calcific changes, and bronchiectasis were also encountered. The presence of two or more typical features was considered as inclusion criteria for diagnosis of active PTB infection. Fibro-calcific and bronchiectatic changes were often presented as sequelae to previous primary PTB infection. Patients with positive culture reports for mycobacteria species other than M. tuberculosis and suspected PTB cases without the above-described HRCT feature were excluded. HRCT features of acute pulmonary infections other than PTB were also excluded.

Thoracic HRCT examination was done with a GE Optima CT660 multi-slice CT scanner (GE Healthcare Japan Corporation, Tokyo) with scanning parameters such as $512 \times 512$ matrix, 1-3 mm collimation, 2-second scan time, $120 \mathrm{kvP}-200 \mathrm{~mA}$, and a bone algorithm at $10 \mathrm{~mm}$ intervals with $35-40 \mathrm{~cm}$ field of view. Contrast-enhanced scanning was not done because it was not indicated.

\section{Results}

Several HRCT features of PTB in elderly patients were recorded (Table 1). Common parenchymal infiltrates were as follows: centrilobular nodules, 'tree-in-bud' pattern densities (Figure 1), macro-nodules (Figure 2), consolidations (Figure 3), cavitary lesions (Figure 3), ground-glass opacities, and miliary nodules (Figure 4). Other associated features were mediastinal adenopathy, pleural effusion, bronchiectasis, and fibro-calcific changes. Features such as centrilobular nodules and branching nodular 'tree-inbud' pattern densities were the characteristic findings of bronchogenic spread mostly seen with post-primary TB infection. Ground-glass opacities were commonly found in areas surrounding nodular lesions. Lobar consolidations of tubercular origin were the result of conglomeration of contiguous segmental consolidations arising from confluent centrilobular opacities. Herein, consolidation was a relatively common finding in the elderly population, which could be attributed to delayed diagnosis of PTB at an advanced stage. Cavitary lesions were detected in two elderly patients with negative culture reports having features mimicking PTB. Walls of cavitary lesions ranged from thin and smooth texture to thick and nodular 
texture. Mediastinal lymph nodes measuring more than $1 \mathrm{~cm}$ in short-axis diameter were considered as adenopathy. Features mimicking PTB, such as fibro-calcific and bronchiectatic changes, were presented by nine cases having negative culture reports.

From a total of 124 (1.0) recorded cases, 108 (0.871) were with features of active PTB infection, while only 16 (0.129) cases had HRCT features mimicking PTB. Furthermore, 106 (0.855) cases had active PTB infection with positive culture test results, and the remaining $18(0.145)$ cases were culture negative for TB infection. Test results of all cases were grouped into four types as TP, TN, FP, and FN cases (Table 2). Thus, there was a mismatch of HRCT results with culture test results. The total of 106 active PTB infection $(\mathrm{TP}+\mathrm{FN})$ cases signifies that the prevalence of active PTB infection was 106 (85.5\%) in the cited population of 124 cases. The sensitivity value is the ability of the HRCT test to detect the active status of the PTB infection, when it is truly present, i.e. it is the probability of a 'positive test result'. Alternately, the specificity value is the ability of the HRCT test to reach at the negative status of the PTB infection (when it is truly absent), i.e. it is the probability of a 'negative test result'. Moreover, the diagnostic accuracy value digitally estimates the accuracy of both HRCT and culture tests together. When the Bayesian concept is applied with the data set (Table 3), several other test statistics were computed for additional probability values, along with corresponding $95 \%$ confidence interval (CI) values for each test statistic (Table 3).

\section{A posteriori probability}

The test statistic, a posteriori probability, the probability value of TB infection to be truly positive, was calculated by the Bayesian formula (Table 3 ). There are two probability values: one $p\left(E_{1} \mid E\right)=0.5617$ (probability of HRCT test for active PTB infection); and the other value is the AUC

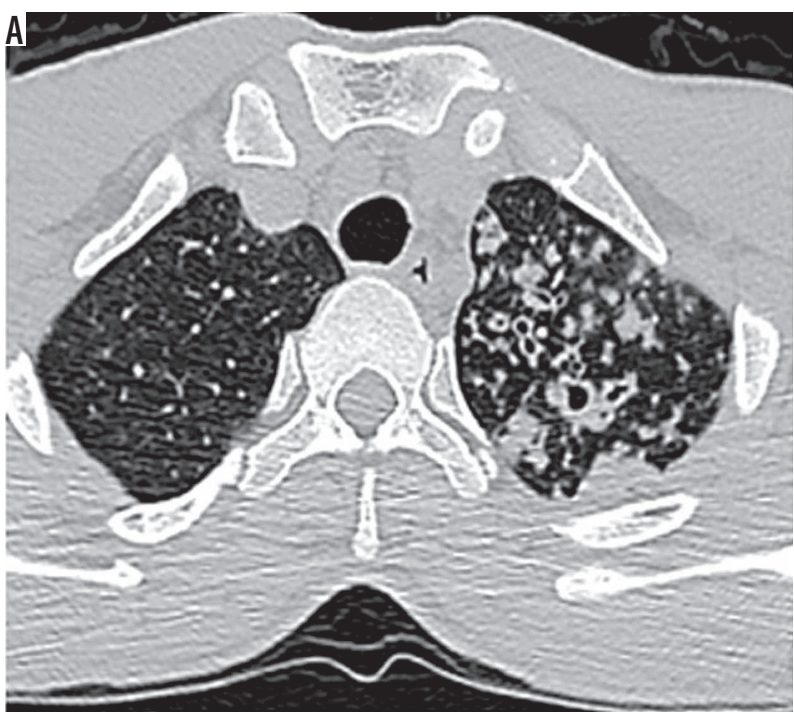

Table 1. Thoracic high-resolution computed tomography (HRCT) features of elderly pulmonary tuberculosis patients

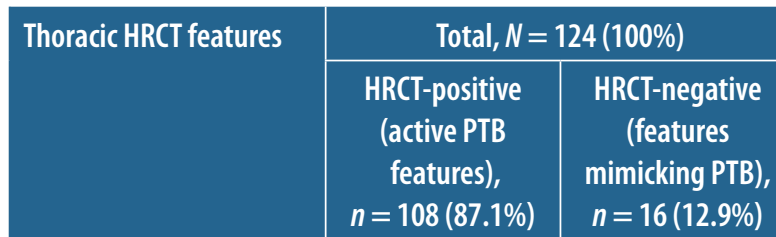

Typical features

\begin{tabular}{|l|c|c|}
\hline Centrilobular nodules & $89(82.3)$ & 0 \\
\hline Tree-in-bud pattern & $86(79.2)$ & 0 \\
\hline Macronodular infiltrates & $84(77.5)$ & 0 \\
\hline Consolidations & $63(58.1)$ & 0 \\
\hline Cavitary lesions & $68(63.2)$ & $6(35.4)$ \\
\hline Ground-glass opacities & $67(61.8)$ & $5(33.3)$ \\
\hline Miliary nodules & $34(31.6)$ & 0 \\
\hline Associated features & & \\
\hline Mediastinal adenopathy & $37(34.2)$ & 0 \\
\hline Pleural effusions & $11(10.5)$ & 0 \\
\hline Bronchiectasis & $58(53.9)$ & $11(68.7)$ \\
\hline Fibro-calcific changes & $38(71.1)$ & $15(91.7)$ \\
\hline
\end{tabular}

value $=0.71$ (from the ROC curve, Figure 5). The mean value of a posteriori probability was calculated as 0.6358 (95\% CI: 0.3107-1.0798) (Table 3).

\section{Discussion}

\section{About the disease}

Cell-mediated immunity (CMI) is the host defence mechanism acting against $\mathrm{TB}$, and it can be profoundly affected by the nutritional status of the patient [6]. Elderly people

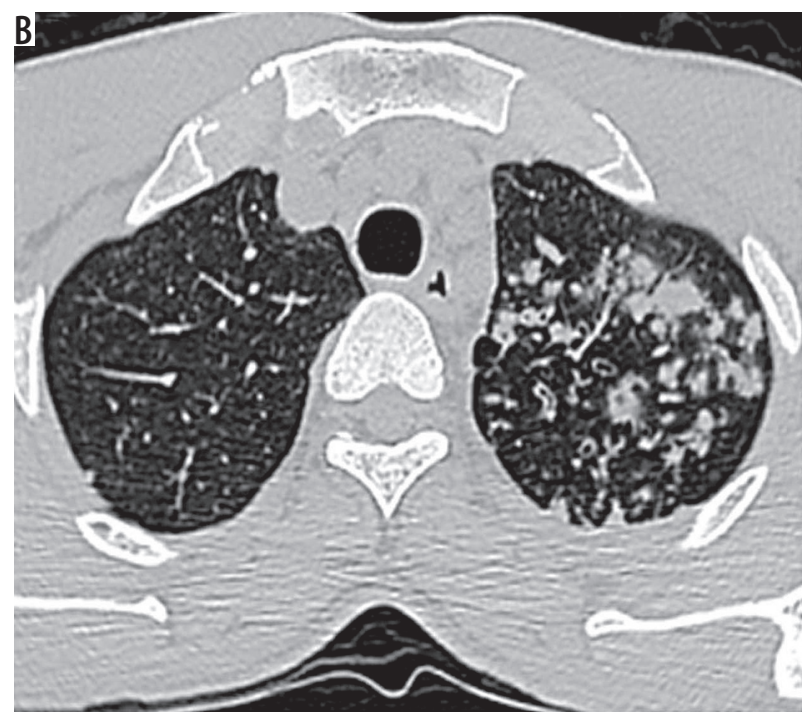

Figure 1. High-resolution computed tomography of lung apices $(A, B)$ in a 66 -year-old female with sputum culture positive for acid fast bacilli showing centrilobular nodules, branching nodular, and linear opacities (tree-in-bud pattern) - typical findings of active pulmonary tuberculosis 

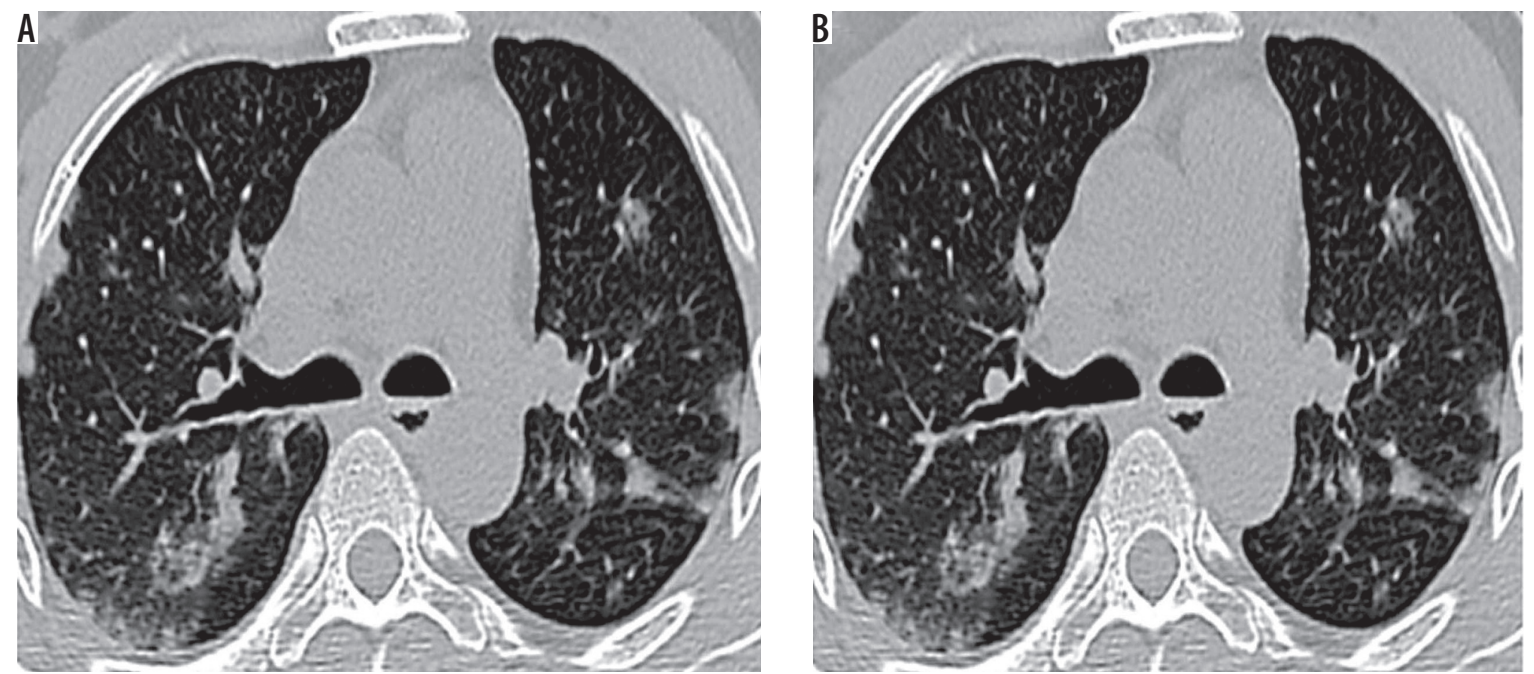

Figure 2. Thoracic high-resolution computed tomography sections at the level of tracheal bifurcation in a 69 -year-old male with sputum culture positive for acid fast bacilli showing (A, B) discrete centrilobular/acinar-lobular infiltrates and sub-pleural macro-nodular densities - typical findings for active pulmonary tuberculosis
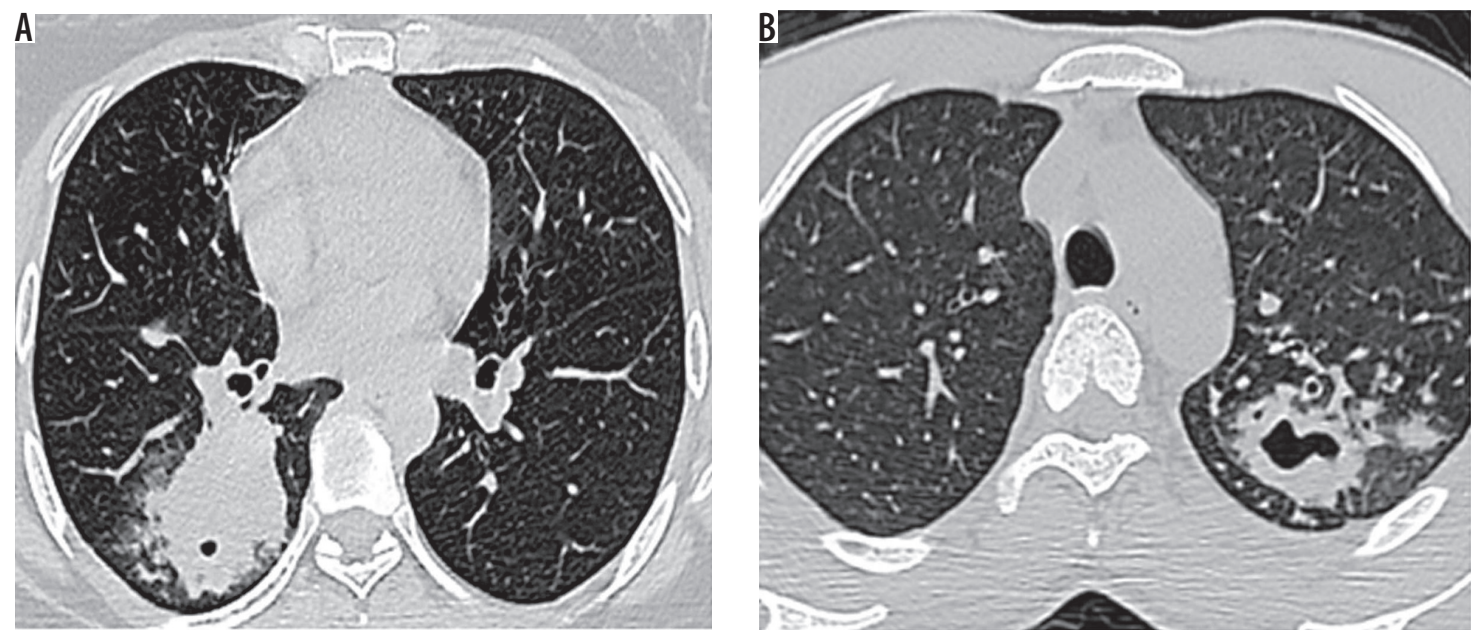

Figure 3. High-resolution computed tomography sections (A) at the level of right lower lung lobe posterior-basal segment showing basal zone predominant airspace consolidation containing a tiny cavity in a 74-year-old male (B) at the level of left upper lung lobe apico-posterior segment showing consolidation and acinus-sized nodules containing few cavities in a 70-year-old male - findings of reactivation pulmonary tuberculosis
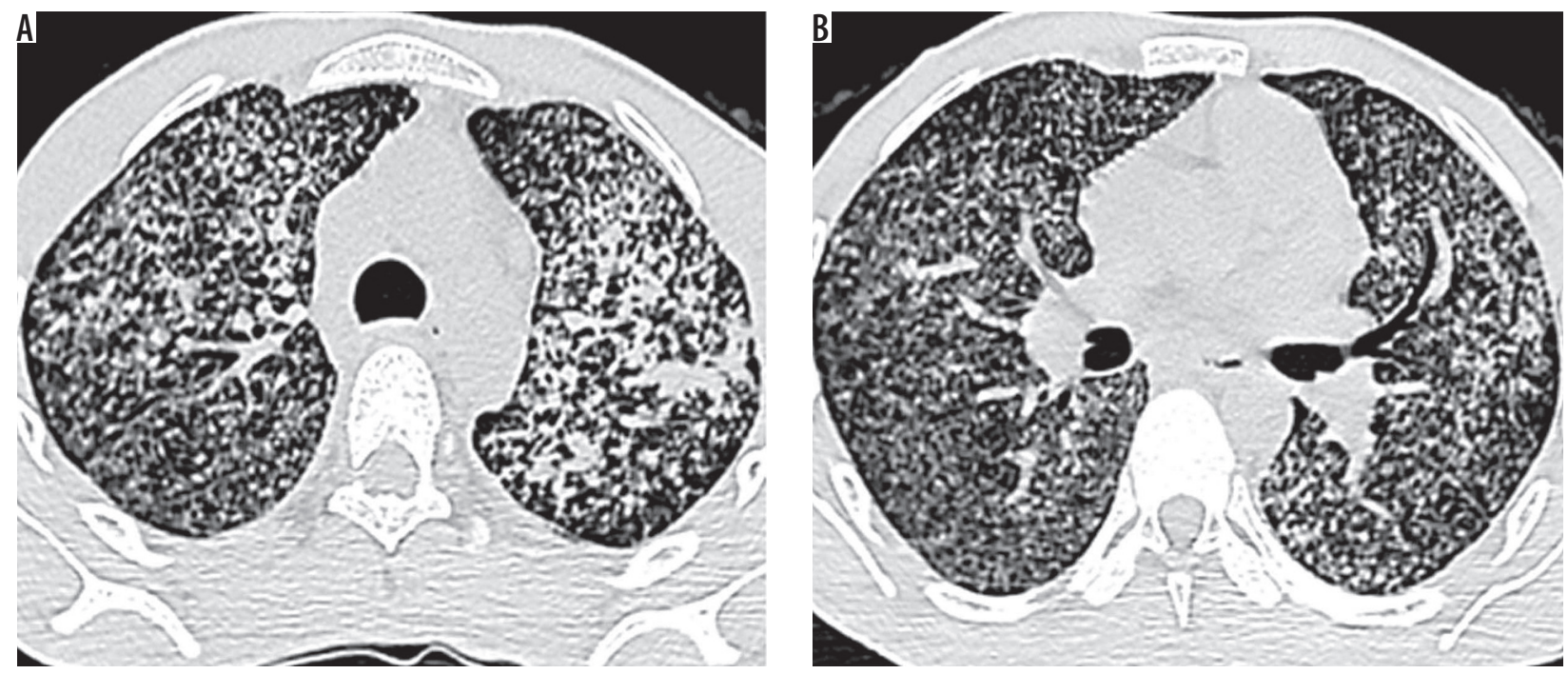

Figure 4. Thoracic high-resolution computed tomography sections in a 66-year-old female with sputum culture positive for acid fast bacilli showing innumerable tiny miliary nodules with a background of patchy ground-glass opacities scattered throughout both lungs. A, B) Sub-pleural and centrilobular miliary nodules as well as nodularity of interlobular septa are noted s/o miliary pattern pulmonary tuberculosis 
Table 2. Generic $2 \times 2$ table with number of cases assigned to active pulmonary tuberculosis (PTB) features and features mimicking PTB, based on thoracic high-resolution computed tomography (HRCT) test against the results of culture test for PTB infection

\begin{tabular}{|l|c|c|c|}
\multirow{2}{*}{ Thoracic HRCT results } & \multicolumn{2}{|c}{ Culture test results } & \multirow{2}{*}{ Total } \\
\cline { 2 - 4 } & Culture-positive* & Culture-negative & $(\mathrm{TP}+\mathrm{FP})=108(0.871)$ \\
\hline HRCT-positive (active PTB features) & $\mathrm{TP}=102(0.823)$ & $\mathrm{FP}=6(0.048)$ & $(\mathrm{FN}+\mathrm{TN})=16(0.129)$ \\
\hline HRCT-negative (features mimicking PTB) & $\mathrm{FN}=4(0.032)$ & $\mathrm{TN}=12(0.097)$ & $N=124(1.0)$ \\
\hline Total & $(\mathrm{TP}+\mathrm{FN})=106(0.855)$ & $(\mathrm{FP}+\mathrm{TN})=18(0.145)$ & $\mathrm{N}$ \\
\hline
\end{tabular}

*Positive for Mycobacterium tuberculosis, TP - 102 cases were true positives (HRCT-positive, culture-positive), FP - 6 cases were false positives (HRCT-positive, culture-negative), $\mathrm{FN}-4$ cases were false negatives (HRCT-negative, culture-positive), and TN -12 samples were true negatives (HRCT-negative, culture-negative), $N$ - population size or total number of cases. Corresponding fraction values are given in parentheses. Prevalence of active PTB infection in the population was 0.855 . FP cases are type I errors, while FN cases are type Il errors.

Table 3. Computed probability values of different Bayesian test statistics in diagnosis of pulmonary tuberculosis

\begin{tabular}{|l|c|c|c|}
\hline Test statistic & Formula & Value & $95 \% \mathrm{Cl}$ \\
\hline Prevalence or a priori probability & (TP + FN)/N & 0.6956 & $0.7560-1.1390$ \\
\hline Sensitivity (true positive rate) & $\mathrm{TP} /(\mathrm{TP}+\mathrm{FN})$ & 0.8125 & $0.8388-1.0612$ \\
\hline Specificity (true negative rate) & $\mathrm{TN} /(\mathrm{FP}+\mathrm{TN})$ & 0.8571 & $0.9139-1.9860$ \\
\hline Diagnostic accuracy & $(\mathrm{TP}+\mathrm{TN}) / \mathrm{N}$ & 0.8260 & $0.8930-1.0010$ \\
\hline Positive predictivity & $\mathrm{TP} /(\mathrm{TP}+\mathrm{FP})$ & 0.9285 & $0.8651-1.0349$ \\
\hline Negative predictivity & $\mathrm{TN} /(\mathrm{FN}+\mathrm{TN})$ & 0.6666 & $0.8996-1.0040$ \\
\hline False positive rate & $\mathrm{FP} /(\mathrm{FP}+\mathrm{TN})=(1-$ specificity) & 0.1429 & $0.7451-1.1549$ \\
\hline False negative rate & $\mathrm{FN} /(\mathrm{TP}+\mathrm{FN})=(1-$ sensitivity) & 0.1875 & $0.7741-1.1259$ \\
\hline Positive likelihood ratio & $\mathrm{sensitivity/(1-specificity)}$ & 5.9975 & $0.7090-1.1910$ \\
\hline Negative likelihood ratio & $(1-\mathrm{sensitivity)/specificity}$ & 0.2187 & $0.7752-1.1248$ \\
\hline A posteriori probability, from computation & $\mathrm{p}(\mathrm{E} 1 / \mathrm{E})$ & 0.5617 & $0.0163-1.1133$ \\
\hline Area under the ROC curve or AUC & & 0.7100 & $0.6051-1.0462$ \\
\hline Mean value of a posteriori probability & & 0.6358 & $0.3107-1.0798$ \\
\hline
\end{tabular}

AUC - area under the ROC curve, $\mathrm{Cl}$ - confidence interval, $\mathrm{FN}$ - false negatives, $\mathrm{FP}$ - false positives, $\mathrm{N}$ - total number of samples, $\mathrm{ROC}$ - receiver operating characteristic curve, $\mathrm{TN}$ - true negatives, TP - true positives

with malnutrition or micronutrient deficiencies are comparatively more prone to this disease with an impaired CMI status. Elderly PTB patients often present widespread lesions as shown by extensive HRCT features leading to poor disease prognosis $[7,8]$. In presence of other diseases such as diabetes, malignancy, and alcoholism, elderly people become more susceptible to PTB from altered mucociliary clearance and decreased pulmonary functions. Biological changes found with aging are integumental barrier disruption and impaired microbial clearance mechanisms [9]. Several chronic debilitating diseases in the elderly along with long-term corticosteroid therapy and few lifestyle factors, viz. poor nutrition, poor environmental factors, smoking, alcohol abuse, etc., usually predispose to reactivation of any dormant TB lesion in the body, and thus it remains responsible for the high prevalence of this disease [10].

A higher occurrence of PTB was seen in the elderly males than in the females corroborating this study, with significantly higher frequencies of co-morbidities, namely, chronic renal failure, and cardiovascular and chronic

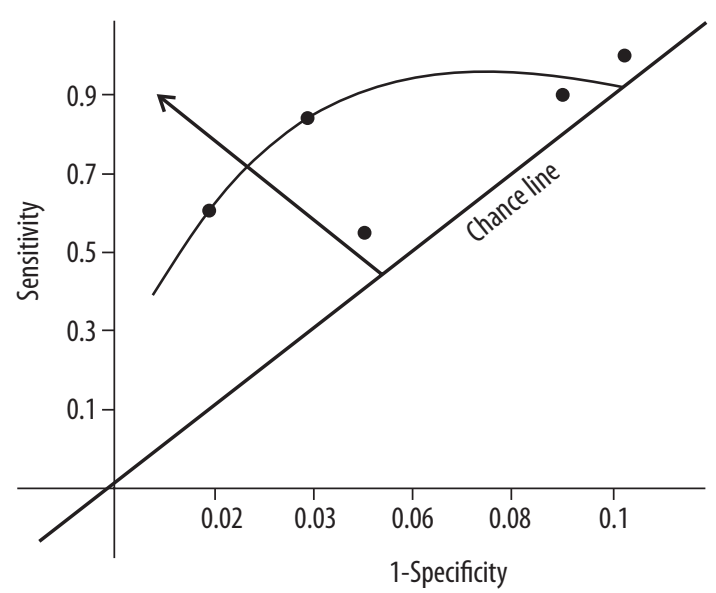

Figure 5. Receiver operating characteristic (ROC) curve for the tuberculosis infection diagnostic data

lung diseases $[1,11]$. Thoracic HRCT plays a major role in the diagnosis of complicated multidrug-resistant (MDR) TB cases, which often show multiple cavities leading to 
expectoration (sputum) of a large number of bacilli into the unaffected lung parenchyma through endo-bronchial spread. While the majority of elderly, active PTB patients present as sputum positive cases, the use of HRCT imaging diagnosis would be prudent to aid in the early diagnosis of the remaining sputum-negative PTB patients [9].

The active PTB lesions in the elderly had a significant effect on mid and lower lung zones due to higher partial pressure of $\mathrm{O}_{2}$ in the lower lung lobe regions $[12,13]$. Upper lung lobe involvement is less likely; elderly patients are more prone to extensive infiltration of bilateral lung parenchyma [14] and are more likely to present consolidations and positive TB culture rates [15]. Another study revealed that lower lung zone predominance of PTB lesions progressively increases with age; whereas, lesions in diabetic patients are more prone to cavitations [16]. Cavities usually harbour large numbers of the mycobacterium, contributing to drug resistance due to limited drug penetration. Present-day TB treatment relies on chemotherapy; nonetheless, surgery may be an adjuvant treatment for MDR TB cases for which HRCT can be a roadmap towards planning the surgical treatment [17].

Elderly PTB is curable if treated early. When non-specific symptoms with radiological non-resolving pulmonary infiltrates are presented, a high index of clinical suspicion for PTB is necessary in the case of elderly patients, to avoid delayed diagnosis and consequent advancement of the disease [18]. Proper management of associated co-morbid diseases, limitations of drug use, minimal invasive procedures, and adequate nutritional support are the cornerstones of clinical control for this disease [9]. The high incidence of mortality from PTB in the elderly could be due to late diagnosis of advanced disease or adverse effects of therapy [19-21].

\section{About the analysis}

All values, except likelihood ratios, are cited herein as fractions of one (Table 3). The prevalence (a priori probability or the pre-test probability or the presence of active PTB infection in the population) value was $69.56 \%$; there were a total of $106(\mathrm{TP}+\mathrm{FN})$ culture positive active PTB infection cases out of a total of $124(\mathrm{~N})$ cases. From incidences of both false cases (FN and FP), it was discernible that each test alone was insufficient for the diagnosis of active PTB infection. Furthermore, both positive predictivity value $(92.85 \%)$ and negative predictivity value $(66.6 \%)$ are computed and are are dependent on the prevalence of the disease; both these values indicated the distinguishing efficiency of the HRCT test (the former for the presence and the later for the absence of active PTB infection) correctly.

Thus, there will not be the ambivalence on the HRCT test, because a clinician inveterately advises a HRCT test in the case of PTB infection, as a part of routine pre-emptive practice. Indeed, it is the most frequently followed method of diagnosis prima facie because it is a quick and non-invasive diagnostic protocol. Moreover, six FP cases (4.8\%) of the total HRCT test results were due to the presence of few active PTB features, misleading the outcome. The assertive statement now would be that the HRCT and culture test had together $56.17 \%$ as the chance of correctly distinguishing a PTB patient for the presence/absence of active infection, as inferred from the a posteriori probability value of 0.5617 .

\section{Limitations of the statistical analysis}

The limitations of this analysis are signposted: 1) high values of sensitivity and specificity are not connected to the prevalence of active PTB infection; 2) in the present study, there was as a low level of error, at 4.8\% (six FP cases), insinuated into the culture test result; thus, it remains the gold standard (verification bias) test.

\section{Conclusions}

High sensitivity and specificity values indicate that the HRCT modality in relation to the gold-standard culture test had increasing importance as a diagnostic tool in differentiating active PTB cases from suspected cases with some HRCT features of other diseases mimicking PTB.

The HRCT test was $69.56-92.85 \%$ efficient in arriving at a positive result with a sample, when its culture test was positive, and it was $66.66-100 \%$ efficient for a negative result, when its culture test result was negative.

Because the sensitivity value of HRCT test is higher, it would be easier to detect the positivity of the disease (active PTB infection) in a population. Therefore, HRCT is a dependable modality for an early, accurate, and honed diagnosis of active PTB.

\section{Conflict of interest}

The authors report no conflict of interest.

\section{References}

1. Okamura K, Nagata N, Kumazoe H, et al. Relationship between computed tomography findings and nutritional status in elderly patients with pulmonary tuberculosis. Intern Med 2011; 50: 1809-1814.
2. Kobashi Y, Mouri K, Fukuda M, et al. Transitional change in the clinical features of pulmonary tuberculosis. Respiration 2008; 75 : 304-309. 
3. Rawat J, Sindhwani G, Juyal R. Clinico-radiological profile of new smear positive pulmonary tuberculosis cases among young adult and elderly people in a tertiary care hospital at Deheradun (Uttarakhand). Indian J Tuberc 2008; 55: 84-90.

4. Sahu N, Padhy RN. Bayesian analysis of high-resolution ultrasonography and guided FNAC in diagnosis of palpable thyroid nodules. Brazilian J Otorhinolaryngol 2016; 84: 20-27.

5. Rosner B. Fundamentals of biostatistics. 5th ed. Duxbury, Pacific Grove, CA 2000.

6. Cegielski JP, McMurray DN. The relationship between malnutrition and tuberculosis: evidence from studies in humans and experimental animals. Int J Tuberc Lung Dis 2004; 8: 286-298.

7. Yabuuchi H, Murayama S, Murakami J, et al. Correlation of immunologic status with high resolution CT and distributions of pulmonary tuberculosis. Acta Radiologica 2002; 43: 44-47.

8. Nagata N, Matsunaga K, Wakamatsu K, et al. The relation between nutritional state of tuberculous patients admitted in tuberculous ward and their outcome at the time of discharge. Kekkaku 2009; 84: 611-616.

9. Rajagopalan S, Yoshikawa TT. Tuberculosis in the elderly. Z Gerontol Geriatr 2000; 33: 374-380.

10. Chan-Yeung M, Chan F, Cheung A, et al. Prevalence of tuberculous infection and active tuberculosis in old age homes in Hong Kong. J Am Geriatr Soc 2006; 54: 1334-1340.

11. Arora VK, Sarin R. Revised national tuberculosis control programme: Indian perspective. Indian J Chest Dis Allied Sci 2000; 42: 21-26.
12. Lee JH, Han DH, Song JW, Chung HS. Diagnostic and therapeutic problems of pulmonary tuberculosis in elderly patients. J Korean Med Sci 2005; 20: 784-789.

13. Wang CS, Chen HC, Yang CJ, et al. The impact of age on the demographic, clinical, radiographic characteristics and treatment outcomes of pulmonary tuberculosis patients in Taiwan. Infection 2008; 36: 335-340.

14. Cantalice Filho JP, Sant Anna CC, Bóia MN. Clinical aspects of pulmonary tuberculosis in elderly patients from a university hospital in Rio de Janeiro, Brazil. J Bras Pneumol 2007; 33: 699-706.

15. Kwon YS, Chi SY, Oh IJ, et al. Clinical characteristics and treatment outcomes of tuberculosis in the elderly: a case control study. BMC Infect Dis 2013; 13: 121.

16. Perez-Guzman C, Torres-Cruz A, Villarreal-velarde H, Vargas MH. Progressive age-related changes in pulmonary tuberculosis images and the effect of diabetes. Am J Respir Crit Care Med 2000; 62: 1738-1740.

17. Takeda S, Maeda H, Hayakawa M, et al. Current surgical intervention for pulmonary tuberculosis. Ann Thorac Surg 2005; 79: 959-963.

18. Sood R. The problem of geriatric tuberculosis. J Indian Acad Clin Med 2000; 5: 156-162.

19. Gaur SN, Dhingra VK, Rajpal S, et al. Tuberculosis in the elderly and their treatment outcome under DOTS. Indian J Tuberc 2004; 51: 83-87.

20. Davies PD. TB in the elderly in industrialised countries. Int J Tuberc Lung Dis 2007; 11: 1157-1159.

21. Packham S. Tuberculosis in the elderly. Gerontology 2001; 47: 175-179. 